\title{
Somatostatinoma in a patient with Neurofibromatosis 1 - Case Report
}

\author{
Rosiek V, Londzin-Olesik M, Zemczak A, Kos-Kudła B
}

Division of Endocrinology, Department of Pathophysiology and Endocrinology, Silesian Medical University, Katowice, Poland

\section{Introduction}

We present a rare case of a 49-year-old patient with neurofibromatosis type $I$ and somatostatin-secreting pancreatic neuroendocrine neoplasm. World literature describes about 30 cases of duodenal somatostatinoma in NFI1 and 1/3 of these cases with metastases to the lymph nodes or liver.

\section{History of Case Report}

In October 2013, a patient was operated on in an emergency situation due to gastrointestinal tract obstruction. During the procedure, an inoperable head of pancreas tumor, closing duodenum was confirmed. A gastrojejuniostomy procedure was performed and specimens from pancreas tumor were taken. No neoplasm tissue was found in the specimens as a result of an histopathological examination.

In June 2014, a core biopsy for pancreas tumor was performed through a controlled CT, which revealed neuroendocrine neoplasm G2 (Fig.1).

Afterwards, on 30th Sept. 2014, the patient was operated on (using Whipple's method).

The diagnosis, i.e. somatostatin producing well-differentiated neuroenocrine neoplasm G2, Ki-67 5\%, was confirmed (positive somatostatin reaction/expression in an immunohistochemical test).

In an abdominal CT on 17th Dec. 2014, local recurrence and metastases to liver and lymph nodes were identified (Fig.2).

${ }^{68} \mathrm{Ga}$ DOTA-TATE PET/CT (Fig.3) and ${ }^{18}$ FDG PET/CT (Fig. 4) were negative.

Stomach aches, weight loss and loose stools (4 per day) since Oct. 2014 have occurred.

Evaluated concentrations of neuroendocrine markers (CgA, insulin, gastrin, serotonin and 5-hydroxyindoleactic acid) remained within the reference ranges. Determination of serum somatostatin level was impossible.

Due to the symptomatic and metastatic character of neuroendocrine neoplasm, the patient was treated with the somatostatin analogue. Clinical improvement (weight stabilization, diarrhea withdrawal) was observed.
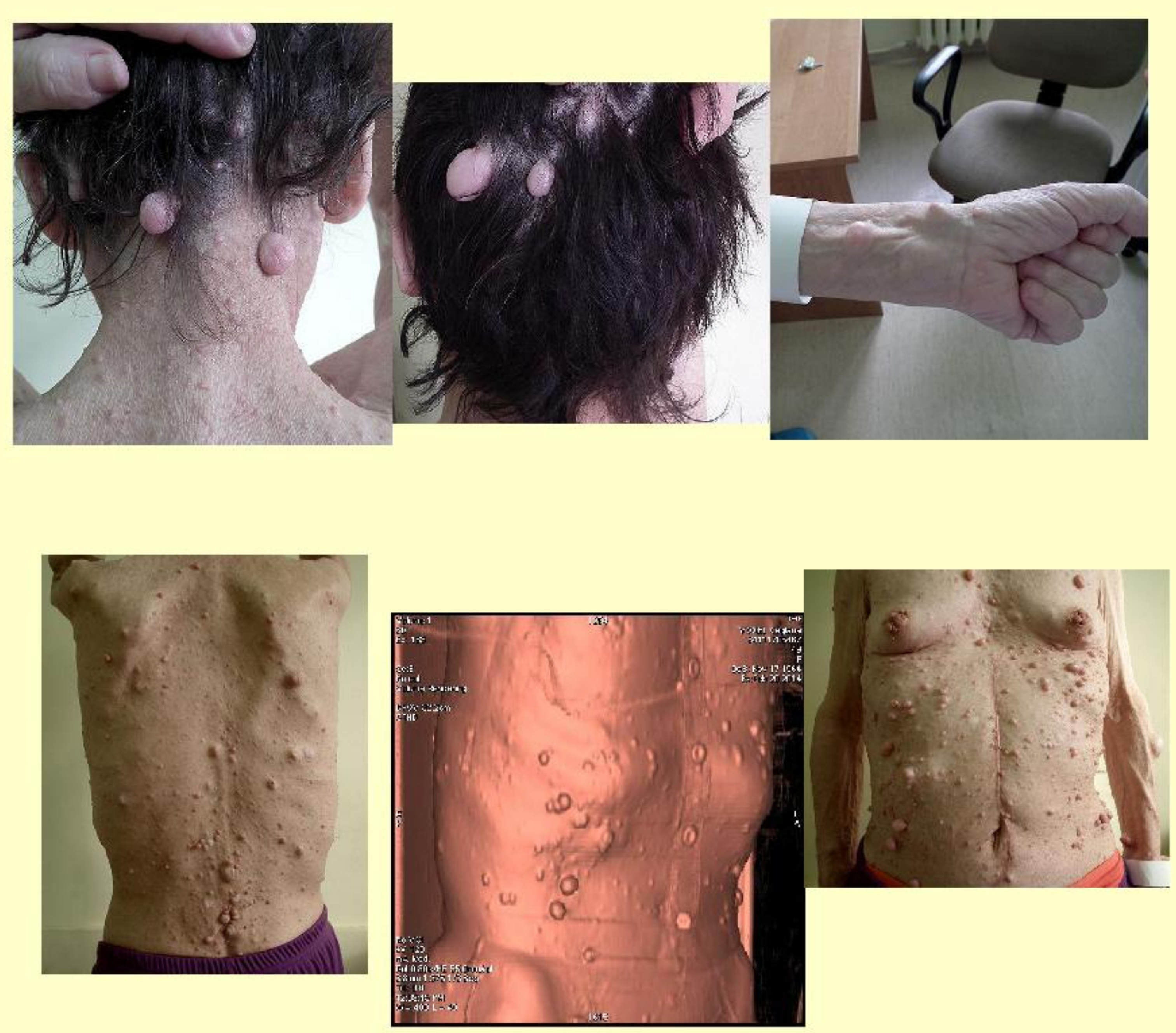
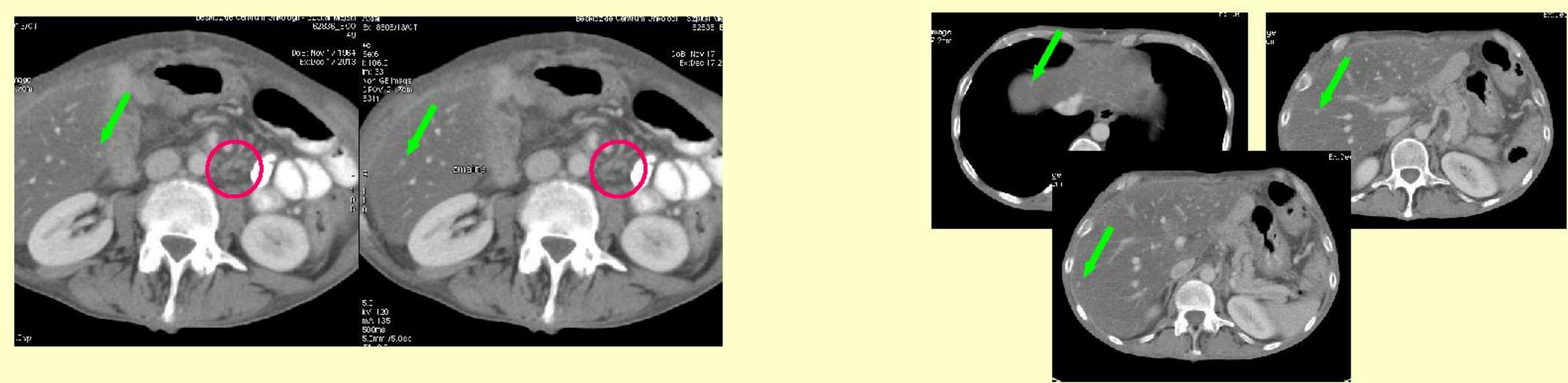

Figure 2. Abdominal CT scan: progressive disease (local recurrence and liver metastases).
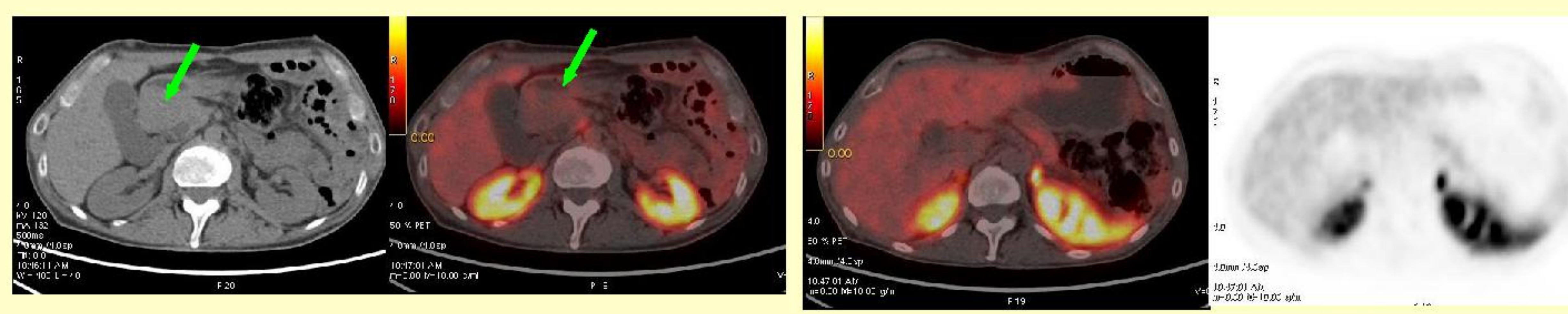

Figure 3. 68Ga DOTA-TATE PET/CT - negative: a pancreatic head tumour measuring $41 \times 37 \mathrm{~mm}$ without overexpression of somatostatin receptors.
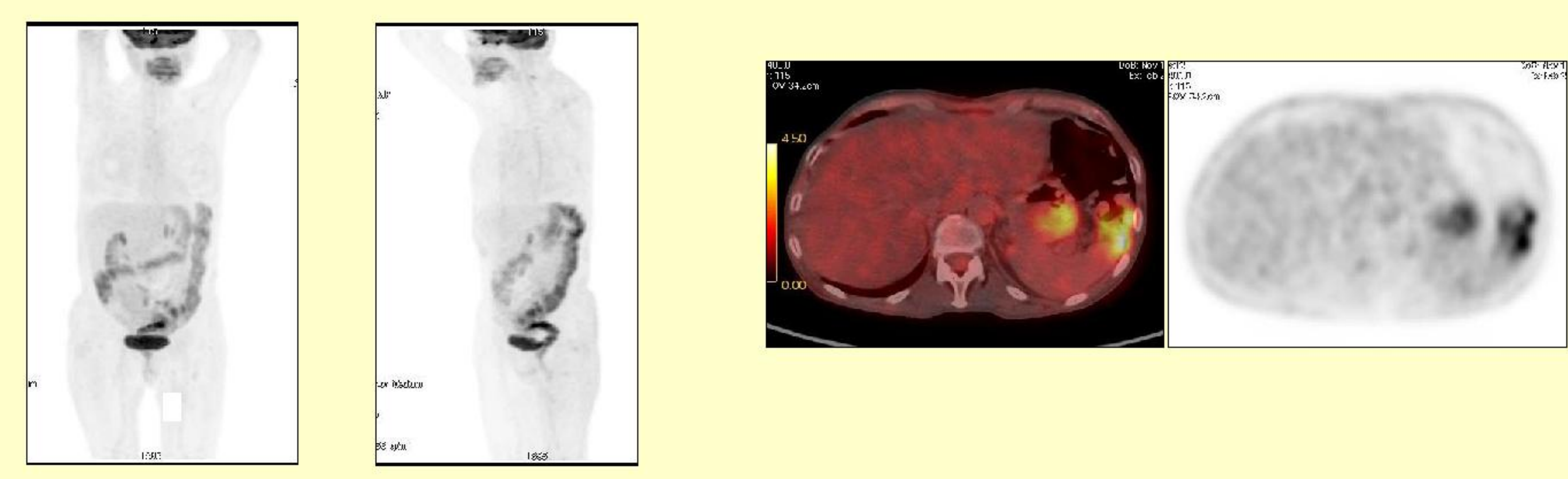

Figure 4. 18FDG PET/CT negative: no foci of increased glucose metabolism.

\section{Conclusion}

Somatostatin-secreting pancreatic neuroendocrine neoplasm occurs very rarely and affects patients with neurofibromatosis type $I$.

Somatostatin analogues in functioning neuroendocrine neoplasm treatment resulted in great effectiveness. 